Bull. Soc. math. France

131 (3), 2003, p. 359-372

\title{
EQUIDISTRIBUTION TOWARDS THE GREEN CURRENT
}

\author{
BY Vincent Guedj
}

\begin{abstract}
Let $f: \mathbb{P}^{k} \rightarrow \mathbb{P}^{k}$ be a dominating rational mapping of first algebraic degree $\lambda \geq 2$. If $S$ is a positive closed current of bidegree $(1,1)$ on $\mathbb{P}^{k}$ with zero Lelong numbers, we show - under a natural dynamical assumption - that the pullbacks $\lambda^{-n}\left(f^{n}\right)^{*} S$ converge to the Green current $T_{f}$. For some families of mappings, we get finer convergence results which allow us to characterize all $f^{*}$-invariant currents.

RÉSUMÉ (Équidistribution vers le courant de Green). - Soit $f: \mathbb{P}^{k} \rightarrow \mathbb{P}^{k}$ une application rationnelle dominante de premier degré algébrique $\lambda \geq 2$. Lorsque $S$ est un courant positif fermé de bidegré $(1,1)$ sur $\mathbb{P}^{k}$ dont les nombres de Lelong sont tous nuls, nous montrons, sous une hypothèse dynamique naturelle, que les pull-backs $\lambda^{-n}\left(f^{n}\right)^{*} S$ convergent vers le courant de Green $T_{f}$. Pour certaines familles d'applications, des résultats de convergence raffinés nous permettent de caractériser tous les courants $f^{*}$-invariants.
\end{abstract}

\section{Introduction}

Let $f: \mathbb{P}^{1} \rightarrow \mathbb{P}^{1}$ be a rational map of degree $\lambda \geq 2$. A celebrated result of Brolin, Lyubich, Freire-Lopez-Mañe asserts that the preimages $\lambda^{-n}\left(f^{n}\right)^{*} \sigma$ of any probability measure $\sigma$ on $\mathbb{P}^{1}$ converge to an invariant measure $\mu_{f}$ as soon as $\sigma\left(\mathcal{E}_{f}\right)=0$, where $\mathcal{E}_{f}$ is a (possibly empty) finite exceptional set. The purpose of this note is to prove similar results in higher dimension.

Texte reçu le 17 janvier 2002, révisé le $1^{\text {er }}$ juillet 2002, accepté le 9 septembre 2002 Vincent Guedj, Laboratoire É. Picard, UMR 5580 Université Paul Sabatier, 118 route de Narbonne, 31400 Toulouse (France) • E-mail : guedj@picard.ups-tlse.fr 2000 Mathematics Subject Classification. - 32H50, 58F23, 58F15.

Key words and phrases. - Green current, holomorphic dynamics, volume estimates. 
Let $f: \mathbb{P}^{k} \rightarrow \mathbb{P}^{k}$ be a rational mapping. It can be written $f=\left[P_{0}: \cdots: P_{k}\right]$ in homogeneous coordinates, where the $P_{j}$ 's are homogeneous polynomials of the same degree $\lambda$ (the first algebraic degree of $f$ ) with no common factor $P_{0} \wedge \cdots \wedge P_{k}=1$. Note that when $k \geq 2, f$ is not necessarily holomorphic: it is not well defined on the indeterminacy set $I_{f}=\bigcap_{j} P_{j}^{-1}(0)$ which is an algebraic subset of $\mathbb{P}^{k}$ of codimension $\geq 2$. There are several ways one can try to generalize the one-dimensional result. Given $Z$ an algebraic subset of $\mathbb{P}^{k}$ of pure codimension $p$, one can ask whether $f^{-n}(Z)$ (properly normalized) converges to an invariant current of bidegree $(p, p)$. In this note we focus on the case $p=1$.

Given $S$ a positive closed current of bidegree $(1,1)$ on $\mathbb{P}^{k}$, we consider

$$
S_{n}:=\lambda^{-n}\left(f^{n}\right)^{*} S .
$$

This is a bounded sequence of positive closed currents of bidegree $(1,1)$ on $\mathbb{P}^{k}$. When $S=\omega$ is the Fubini-Study Kähler form, it was proved by Sibony [19] that $\left(\omega_{n}\right)$ converges to an invariant Green current $T_{f}$. On the other hand Russakovskii and Shiffman have shown [18] that $[H]_{n}-\omega_{n} \rightarrow 0$ for almost every hyperplane $H$ of $\mathbb{P}^{k}$. Our main result interpolates between these two extreme cases.

TheOREM 0.1. - Let $f: \mathbb{P}^{k} \rightarrow \mathbb{P}^{k}$ be a dominating rational mapping with $\lambda \geq 2$. Assume there exists an invariant probability measure $\mu$ such that $\log \left|J_{F S}(f)\right| \in L^{1}(\mu)$. Let $S$ be a positive closed current of bidegree $(1,1)$ and unit mass on $\mathbb{P}^{k}$. If $\nu(S, p)=0$ for all $p \in \mathbb{P}^{k}$, then

$$
\frac{1}{\lambda^{n}}\left(f^{n}\right)^{*} S \longrightarrow T_{f} \text { in the weak sense of currents. }
$$

Here $\nu(S, p)$ denotes the Lelong number of $S$ at point $p$ and $J_{F S}(f)$ denotes the jacobian of $f$ with respect to the Fubini-Study volume form $\omega^{k}$. Similar (weaker) results were previously obtained for Hénon mappings [1], [8], birational mappings [6], and holomorphic endomorphisms of $\mathbb{P}^{k}[9],[19],[7]$.

Although Theorem 0.1 does not imply directly Russakovskii-Shiffman's result, the proof shows one essentially has to assume $\sup _{p \in \mathbb{P}^{k} \backslash E} \nu\left(S_{n}, p\right) \rightarrow 0$, where $E$ is some (possibly empty) exceptional set (see Theorem 1.4). The key ingredients of the proof are: a pluripotential estimate of the volume of sublevel sets of a quasiplurisubharmonic function [16] and a dynamical estimate on the decreasing of volumes under iteration (Theorem 1.2). Note that all the volumes are computed with respect to the Fubini-Study volume form $\omega^{k}$.

We prove the volume estimates and our main result in Section 1. We give refinements of the latter in Section 2 in case $f$ is an holomorphic endomorphism of $\mathbb{P}^{k}$ (Section 2.1) or a special type of polynomial endomorphism of $\mathbb{C}^{k}$ (Section 2.2). This allows us to characterize every $f^{*}$-invariant current. Such equidistribution results should be understood as strong ergodic properties of

TOME $131-2003-\mathrm{N}^{\mathrm{O}} 3$ 
the Green current $T_{f}$. In dimension 1 indeed this implies that $T_{f}$ is strongly mixing (see Theorem VIII.22 in [2]). For the reader's convenience we recall in an Appendix compactness criteria for families of qpsh functions. They are the higher dimensional counterparts of Montel's Theorem.

Acknowledgement. — We thank Ahmed Zeriahi for several useful conversations concerning this article. We also thank the referee for reading the paper carefully and making helpful comments.

\section{Equidistribution of pullbacks of currents}

Let $f: \mathbb{P}^{k} \rightarrow \mathbb{P}^{k}$ be a rational mapping with first algebraic degree $\lambda \geq 2$. We always assume $f$ is dominating, i.e. its jacobian does not vanish identically in any coordinate chart. It follows that a generic point has $d_{t}(f)$ well defined preimages by $f$. Note that $d_{t}(f)=\lambda$ when $k=1$ but these two degrees differ in general when $k \geq 2$.

Let $\omega$ denote the Fubini-Study Kähler form on $\mathbb{P}^{k}$. The smooth form $f^{*} \omega$ is well defined in $\mathbb{P}^{k} \backslash I_{f}$ and extends trivially through $I_{f}$ as a positive closed current of bidegree $(1,1)$ and mass $\left\|f^{*} \omega\right\|=\int_{\mathbb{P}^{k}} f^{*} \omega \wedge \omega^{k-1}=\lambda$. So $\lambda^{-1} f^{*} \omega$ is cohomologous to $\omega$. Since $\mathbb{P}^{k}$ is Kähler, this can be written

$$
\lambda^{-1} f^{*} \omega=\omega+\operatorname{dd}^{c} G,
$$

where $G \in L^{1}\left(\mathbb{P}^{k}\right)$ (see [11, p. 149]). The function $G$ is "quasiplurisubharmonic" (qpsh): it is locally given as the sum of a psh function (a local potential of $\lambda^{-1} f^{*} \omega \geq 0$ ) and a smooth function (a local potential of $-\omega$ ). In particular it is bounded from above on $\mathbb{P}^{k}$ : replacing $G$ by $G-C_{0}$, we can therefore assume $G \leq 0$. Sibony [19] has shown that the decreasing sequence of qpsh functions

$$
G_{n}:=\sum_{j=0}^{n-1} \frac{1}{\lambda^{j}} G \circ f^{j}
$$

converges in $L^{1}\left(\mathbb{P}^{k}\right)$ to a qpsh function $G_{\infty} \in L^{1}\left(\mathbb{P}^{k}\right)$. This shows that $\lambda^{-n}\left(f^{n}\right)^{*} \omega$ converges in the weak sense of (positive) currents to the so called Green current $T_{f} \geq 0$ which satisfies $f^{*} T_{f}=\lambda T_{f}$.

A natural question is then to look at the convergence of $S_{n}:=\lambda^{-n}\left(f^{n}\right)^{*} S$, where $S$ is now any positive closed current of bidegree $(1,1)$ and unit mass on $\mathbb{P}^{k}$. When $S=[H]$ is the current of integration along an hyperplane of $\mathbb{P}^{k}$, it was shown by Russakovskii and Shiffman [18] that $[H]_{n} \rightarrow T_{f}$ for every $H$ outside some pluripolar set $\mathcal{E} \subset\left(\mathbb{P}^{k}\right)^{*}$. In order to prove convergence of $S_{n}$ for more general currents $S$, we first need to get control on the decreasing of volumes under iteration. 
1.1. Volume estimates. - Let $f: \mathbb{P}^{k} \rightarrow \mathbb{P}^{k}$ be a dominating rational mapping with $\lambda \geq 2$. Let $J_{F S}(f)$ denote its jacobian with respect to the Fubini-Study Kähler volume form. It is defined by

$$
f^{*} \omega^{k}=\left|J_{F S}(f)\right|^{2} \omega^{k} .
$$

Proposition 1.1. - Fix $B$ an open subset of $\mathbb{P}^{k}$ and $\delta_{0}>0$. There exists $C_{0}>0$ such that for every open subset $\Omega$ of $\mathbb{P}^{k}$ with $\operatorname{vol}(\Omega) \geq \delta_{0}$,

$$
\operatorname{vol}\left(f^{n}(\Omega)\right) \geq\left(C_{0}\right)^{\lambda^{n}} \exp \left(\frac{1}{\operatorname{vol}(B) \operatorname{vol}(\Omega)} \int_{B} \log \left|J_{F S}\left(f^{n}\right)\right|^{2} \omega^{k}\right)
$$

for all $n \in \mathbb{N}$.

Proof. — Fix $\mathbb{C}^{k}$ an affine chart of $\mathbb{P}^{k}$. We have

$$
f=\left(P_{1} / P_{0}, \ldots, P_{k} / P_{0}\right)
$$

in $\mathbb{C}^{k}$ where the $P_{j}$ 's are polynomials of degree $\leq \lambda=\delta_{1}(f)$. Since $\omega=$ $\mathrm{dd}^{c} \frac{1}{2} \log \left[1+\|z\|^{2}\right]$ in $\mathbb{C}^{k}$, we get

$$
\omega^{k}(z)=\left(1+\|z\|^{2}\right)^{-(k+1)} \mathrm{d} V,
$$

where $\mathrm{d} V$ denotes the euclidean volume form in $\mathbb{C}^{k}$. Therefore

$$
\left|J_{F S}(f)\right|^{2}=\left|J_{\text {eucl }}(f)\right|^{2}\left(\frac{1+\|z\|^{2}}{1+\|f(z)\|^{2}}\right)^{k+1} .
$$

We infer

$$
\log \left|J_{F S}(f)\right|=u-v,
$$

where $u, v$ are qpsh functions such that $\operatorname{dd}^{c} u, \operatorname{dd}^{c} v \geq-2 \lambda k \omega$. Let $\Omega$ be an open subset of $\mathbb{P}^{k}$. We have

$$
\operatorname{vol}\left(f^{n}(\Omega)\right)=\int_{f^{n}(\Omega)} \omega^{k} \geq \frac{1}{d_{t}(f)^{n}} \int_{\Omega}\left|J_{F S}\left(f^{n}\right)\right|^{2} \omega^{k},
$$

where the inequality follows from the change of variable formula. The concavity of the logarithm yields

$$
\operatorname{vol}\left(f^{n}(\Omega)\right) \geq \frac{\operatorname{vol}(\Omega)}{d_{t}(f)^{n}} \exp \left[\frac{2 D_{n}}{\operatorname{vol}(\Omega)} \int_{\Omega} \frac{1}{D_{n}}\left(u_{n}-v_{n}\right) \omega^{k}\right],
$$

where

$$
\log \left|J_{F S}\left(f^{n}\right)\right|=u_{n}-v_{n}
$$

with $\mathrm{dd}^{c} u_{n}, \mathrm{dd}^{c} v_{n} \geq-D_{n} \omega, D_{n} \leq 2 \lambda^{n} k$. Observe that we can decompose

$$
\frac{1}{D_{n}} \log \left|J_{F S}\left(f^{n}\right)\right|=\varphi_{n}-\psi_{n}+\frac{1}{\operatorname{vol}(B)} \int_{B} \frac{1}{D_{n}} \log \left|J_{F S}\left(f^{n}\right)\right| \omega^{k},
$$

TOME $131-2003-\mathrm{N}^{\mathrm{O}} 3$ 
where

$$
\begin{aligned}
& \varphi_{n}=D_{n}^{-1} u_{n}-\log \|z\|-\frac{1}{\operatorname{vol}(B)} \int_{B}\left(D_{n}^{-1} u_{n}-\log \|z\|\right) \omega^{k}, \\
& \psi_{n}=D_{n}^{-1} v_{n}-\log \|z\|-\frac{1}{\operatorname{vol}(B)} \int_{B}\left(D_{n}^{-1} v_{n}-\log \|z\|\right) \omega^{k} .
\end{aligned}
$$

The functions $\varphi_{n}, \psi_{n}$ are quasiplurisubharmonic on $\mathbb{P}^{k}\left(\operatorname{dd}^{c} \varphi_{n}, \mathrm{dd}^{c} \psi_{n} \geq-\omega\right)$ with $\int_{B} \varphi_{n}=\int_{B} \psi_{n}=0$. It follows therefore from Proposition 3.2 (Appendix) that they belong to a compact family of qpsh functions, so there exists $C_{\Omega} \in \mathbb{R}$ such that $\int_{\Omega}\left(\varphi_{n}-\psi_{n}\right) \omega^{k} \geq C_{\Omega}$, for all $n \in \mathbb{N}$. Since $D_{n} \leq 2 k \lambda^{n}$, this yields the desired inequality.

It remains to get a lower bound on $\int_{B} \log \left|J_{F S}\left(f^{n}\right)\right| \omega^{k}$, where $B$ is an open subset which we may fix as we like.

THEOREM 1.2. - Let $f: \mathbb{P}^{k} \rightarrow \mathbb{P}^{k}$ be a dominating rational mapping with $\lambda \geq 2$. Assume there exists an invariant probability measure $\mu$ such that $\log \left|J_{F S}(f)\right| \in L^{1}(\mu)$. Fix $\delta_{0}>0$. Then there exists $C_{0}>0$ such that for every open subset $\Omega$ of $\mathbb{P}^{k}$ with $\operatorname{vol}(\Omega) \geq \delta_{0}$,

$$
\operatorname{vol}\left(f^{n}(\Omega)\right) \geq C_{0}^{\lambda^{n}}, \quad \forall n \in \mathbb{N} .
$$

Proof. - Using Proposition 1.1, it is sufficient to find $M>0$ such that for all $n$, $\int_{B} \log \left|J_{F S}\left(f^{n}\right)\right| \omega^{k} \geq-M \lambda^{n}$. We take here $B=\mathbb{P}^{k}$ (but other normalisations could be useful, see Remark 1.3 below).

We decompose $\lambda^{-1} \log \left|J_{F S}(f)\right|=u-v+C$, where $u, v$ are qpsh functions $\left(\operatorname{dd}^{c} u, \operatorname{dd}^{c} v \geq-\omega\right)$ such that $\sup _{\mathbb{P}^{k}} u=\sup _{\mathbb{P}^{k}} v=0$ and $C \in \mathbb{R}$. Thus we get

$$
\frac{1}{\lambda^{n}} \log \left|J_{F S}\left(f^{n}\right)\right|=\frac{1}{\lambda^{n}} \sum_{j=0}^{n-1} \log \left|J_{F S}(f) \circ f^{j}\right| \geq \sum_{j=0}^{n-1} \frac{1}{\lambda^{n-j}} u_{j}+\frac{n}{\lambda^{n}} C,
$$

where $u_{j}:=\lambda^{-j} u \circ f^{j}$. It is therefore sufficient to get a uniform lower bound on $\int_{\mathbb{P}^{k}} u_{j} \omega^{k}$. This is a consequence of the fact that $\left(u_{j}\right)$ is relatively compact in $L^{1}\left(\mathbb{P}^{k}\right)$. Indeed $\mathrm{dd}^{c} u_{j} \geq-\lambda^{-j}\left(f^{j}\right)^{*} \omega$, so $u_{j}+G_{j}$ is qpsh. By Lemma 3.1 (Appendix), the sequence $\left(u_{j}+G_{j}\right)$ is either relatively compact or uniformly converges to $-\infty$. Since $G_{j} \rightarrow G_{\infty} \in L^{1}\left(\mathbb{P}^{k}\right)$, the sequence $\left(u_{j}\right)$ is either relatively compact or converges to $-\infty$. But the latter can not happen since $u \in L^{1}(\mu)$ and $\int u_{j} \mathrm{~d} \mu=\lambda^{-j} \int u \mathrm{~d} \mu \rightarrow 0$. The desired control on $\int_{\mathbb{P}^{k}} \log \left|J_{F S}\left(f^{n}\right)\right| \omega^{k}$ follows.

REMARK 1.3. - The assumption on the existence of $\mu$ is natural in our dynamical context. Observe that it is satisfied if e.g. there exists a non critical periodic point.

Other assumptions could be made to obtain the final lower bound on $\int_{B} \log \left|J_{F S}\left(f^{n}\right)\right| \omega^{k}$. If $f_{\mid \mathbb{C}^{k}}$ is polynomial, it is enough to assume that

BULletin DE LA SOCIÉtÉ MATHÉMATIQUE DE FRANCE 
$\sup _{B}\left|J_{\text {eucl }}\left(f^{n}\right)\right| \geq \alpha^{\lambda^{n}}$ for some relatively compact open subset $B$ of $\mathbb{C}^{k}$. This holds in particular when $f$ is a polynomial automorphism of $\mathbb{C}^{k}$.

1.2. Equidistribution of pullbacks. - A major difficulty in higher dimensional complex dynamics lies in the presence of points of indeterminacy and in the difficulty of analyzing the dynamics near

$$
I_{f}^{\infty}:=\bigcup_{n \geq 0} I_{f^{n}} .
$$

Following Fornaess and Sibony [9], a rational mapping $f: \mathbb{P}^{k} \rightarrow \mathbb{P}^{k}$ is said to be normal if for every $p \in \mathbb{P}^{k} \backslash \overline{I_{f}^{\infty}}$, there exists a neighborhood $W$ of $p$ and $V$ of $\overline{I_{f}^{\infty}}$ such that $f^{n}(W) \cap V=\varnothing$ for all $n \geq 0$. Note that the condition is empty if $\overline{I_{f}^{\infty}}=\mathbb{P}^{k}$, so one usually assumes further that $\overline{I_{f}^{\infty}}$ is "small", e.g. $\operatorname{vol}\left(\overline{I_{f}^{\infty}}\right)=0$. Examples of such mappings include holomorphic endomorphisms of $\mathbb{P}^{k}$ (for which $I_{f}=I_{f}^{\infty}=\varnothing$ ) or some polynomial endomorphisms of $\mathbb{C}^{k}$ with small topological degree (e.g. Hénon mappings in $\mathbb{C}^{2}$, see other examples in [12], [13] and Section 2.2 below).

THEOREM 1.4. - Let $f: \mathbb{P}^{k} \rightarrow \mathbb{P}^{k}$ be a dominating rational mapping with $\lambda \geq 2$. Assume there exists an invariant probability measure $\mu$ on $\mathbb{P}^{k}$ such that $\log \left|J_{F S}(f)\right| \in L^{1}(\mu)$. Let $S$ be a positive closed current of bidegree $(1,1)$ and unit mass on $\mathbb{P}^{k}$. If $\nu(S, p)=0$ for all $p \in \mathbb{P}^{k}$, then

$$
S_{n}:=\frac{1}{\lambda^{n}}\left(f^{n}\right)^{*} S \longrightarrow T_{f} .
$$

Moreover if $f$ is normal with $\operatorname{vol}\left(\overline{I_{f}^{\infty}}\right)=0$ then $S_{n} \rightarrow T_{f}$ if and only if $\nu\left(S_{n}, p\right) \rightarrow 0$ uniformly on compact subsets of $\mathbb{P}^{k} \backslash \overline{I_{f}^{\infty}}$.

Proof. - Write $S=\omega+\mathrm{dd}^{c} u$, where $u \leq 0$ is quasiplurisubharmonic on $\mathbb{P}^{k}$. Then $\lambda^{-n}\left(f^{n}\right)^{*}(S-\omega)=\operatorname{dd}^{c} u_{n}$, where $u_{n}=\lambda^{-n} u \circ f^{n} \leq 0$. So we need to show that $u_{n} \rightarrow 0$ in $L^{1}\left(\mathbb{P}^{k}\right)$.

Observe that $\operatorname{dd}^{c}\left(u_{n}+G_{n}\right) \geq-\omega$, so $\left(u_{n}\right)$ is either relatively compact or uniformly converges to $-\infty$ (Lemma 3.1$)$. It is therefore sufficient to prove that for all $\varepsilon>0, \operatorname{vol}\left(\Omega_{n}^{\varepsilon}\right) \rightarrow 0$, where

$$
\Omega_{n}^{\varepsilon}:=\left\{p \in \mathbb{P}^{k} ; \frac{1}{\lambda^{n}} u \circ f^{n}(p)<-\varepsilon\right\} .
$$

Assume on the contrary that $\operatorname{vol}\left(\Omega_{n_{i}}^{\varepsilon}\right) \geq \delta_{0}$ for some fixed $\varepsilon, \delta_{0}>0$ and $n_{i} \rightarrow+\infty$. Observe that

$$
f^{n_{i}}\left(\Omega_{n_{i}}^{\varepsilon}\right) \subset\left\{p \in \mathbb{P}^{k} ; u(p)<-\varepsilon \lambda^{n_{i}}\right\} .
$$

If $\nu(S, p)=0$ for all $p \in \mathbb{P}^{k}$, it follows from Skoda's integrability Theorem (see Theorem 3.1 in [16]) that for every $A>0$, there exists $C_{A}>0$ such that

$$
\operatorname{vol}\left(f^{n_{i}}\left(\Omega_{n_{i}}^{\varepsilon}\right)\right) \leq C_{A} \exp \left(-A \varepsilon \lambda^{n_{i}}\right) .
$$

TOME $131-2003-\mathrm{N}^{\mathrm{O}} 3$ 
On the other hand, since $\operatorname{vol}\left(\Omega_{n_{i}}^{\varepsilon}\right) \geq \delta_{0}>0$, there follows from Theorem 1.2 that there exists $C_{0}>0$ such that

$$
\operatorname{vol}\left(f^{n_{i}}\left(\Omega_{n_{i}}^{\varepsilon}\right)\right) \geq C_{0}^{\lambda^{n_{i}}}, \text { for all } i \in \mathbb{N} .
$$

Taking $A>-\log C_{0}$ yields a contradiction.

Assume now $f$ is normal. It was proved by Favre [5] that $\nu\left(T_{f}, p\right)=0$ for all $p \in \mathbb{P}^{k} \backslash I_{f}^{\infty}$. Therefore it is necessary, for $S_{n}$ to converge to $T_{f}$, that for every open neighborhood $V$ of $\overline{I_{f}^{\infty}}, \sup _{p \in \mathbb{P}^{k} \backslash V} \nu\left(S_{n}, p\right) \rightarrow 0$. This is because $(S, p) \mapsto \nu(S, p)$ is upper semi-continuous (u.s.c.). Assume it is the case. Fix $W$ a relatively compact open subset of $\mathbb{P}^{k} \backslash \overline{I_{f}^{\infty}}$. Since $\operatorname{Vol}\left(\overline{I_{f}^{\infty}}\right)=0$, it is sufficient to prove that $u_{n} \rightarrow 0$ on every such $W$. Since $f$ is normal, we can fix $V$ an open neighborhood of $\overline{I_{f}^{\infty}}$ such that $V \cap f^{n}(W)=\varnothing$, for all $n \geq 0$. We need to prove that $\operatorname{vol}\left(W \cap \Omega_{n}^{\varepsilon}\right) \rightarrow 0$. Now

$$
f^{n}\left(W \cap \Omega_{n}^{\varepsilon}\right) \subset\left\{p \in \mathbb{P}^{k} \backslash V ; u(p)<-\varepsilon \lambda^{n}\right\},
$$

so the previous proof applies if $\sup _{p \in \mathbb{P}^{k} \backslash V} \nu(S, p)$ is small enough. When $\sup _{p \in \mathbb{P}^{k} \backslash V} \nu(S, p)$ is not small enough, we replace $S$ by $S_{N_{0}}, N_{0} \gg 1$.

\section{Invariant currents}

It is an interesting problem to characterize all positive closed currents $S$ of bidegree $(1,1)$ on $\mathbb{P}^{k}$ such that $f^{*} S=\lambda S$. This can be done by using our equidistribution result (Theorem 1.4). We illustrate this on two families of mappings.

2.1. Holomorphic endomorphisms of $\mathbb{P}^{k}$. - We assume here $f: \mathbb{P}^{k} \rightarrow \mathbb{P}^{k}$ is holomorphic, i.e. $I_{f}=\varnothing$. In this case the construction of the Green current $T_{f}$ is due to Hubbard and Papadopol [15]: $G$ is smooth on $\mathbb{P}^{k}$, so $\left(G_{n}\right)$ uniformly converges to $G_{\infty}$ which is henceforth continuous (see $(*)$ ).

Since the Green current $T_{f}$ has continuous potential, all its Lelong numbers are 0. Moreover, it follows from the work of Bedford and Taylor that the measure $\mu_{f}:=T_{f}^{k}$ is well defined. The measure $\mu_{f}$ is invariant and every qpsh function is $\mu_{f}$ integrable (as follows from the Chern-Levine-Nirenberg inequalities, see the Appendix in [19]). Therefore $f$ satisfies the assumptions of Theorem 1.4. Given $S$ a positive closed current of bidegree $(1,1)$ and unit mass on $\mathbb{P}^{k}$, Theorem 1.4 reads here

$$
\frac{1}{\lambda^{n}}\left(f^{n}\right)^{*} S \rightarrow T_{f} \Longleftrightarrow \sup _{p \in \mathbb{P}^{k}} \nu\left(\lambda^{-n}\left(f^{n}\right)^{*} S, p\right) \rightarrow 0
$$

It remains to understand the behavior of Lelong numbers under iteration. Since $f$ is proper, one easily gets

$$
\nu\left(\left(f^{n}\right)^{*} S, p\right) \leq d\left(f^{n}, p\right) \nu\left(S, f^{n}(p)\right),
$$

BULLETIN DE LA SOCiÉtÉ MATHÉMATIQUE DE FRANCE 
where $d\left(f^{n}, p\right)$ denotes the local topological degree of $f^{n}$ at $p, d\left(f^{n}, p\right)=$ $\Pi_{j=0}^{n-1} d\left(f, f^{j}(p)\right)$. So we are done if $d\left(f^{n}, p\right)=o\left(\lambda^{n}\right)$.

Analyzing the behavior of $d\left(f^{n}, p\right)$ is quite easy in dimension 1 as shows the following elementary lemma whose proof is left to the reader.

Lemma 2.1. - Let $f: \mathbb{P}^{1} \rightarrow \mathbb{P}^{1}$ be a rational map of degree $\lambda \geq 2$. Set

$$
\mathcal{E}_{f}:=\left\{p \in \mathbb{P}^{1} ; d(f, p)=d(f, f(p))=d\left(f, f^{2}(p)\right)=\lambda\right\} .
$$

Then either

- $\mathcal{E}_{f}$ is empty, or

- $\mathcal{E}_{f}=1$ point, $f$ is conjugate to a polynomial, or else

- $\mathcal{E}_{f}=2$ points, $f$ is conjugate to $z^{\lambda}$ or $z^{-\lambda}$.

Combining this with Theorem 1.4 yields the following celebrated result of Brolin [3], Lyubich [17] and Freire-Lopez-Mañe [10]. Note that positive closed currents of bidegree $(1,1)$ and unit mass are simply probability measures on $\mathbb{P}^{1}$.

Theorem 2.2. - Let $f: \mathbb{P}^{1} \rightarrow \mathbb{P}^{1}$ be a rational map of degree $\lambda \geq 2$. Let $\sigma$ be a probability measure on $\mathbb{P}^{1}$. Then

$$
\frac{1}{\lambda^{n}}\left(f^{n}\right)^{*} \sigma \rightarrow T_{f} \Longleftrightarrow \sigma\left(\mathcal{E}_{f}\right)=0 .
$$

When $k \geq 2$ the "crude" estimate $d\left(f^{n}, p\right) \leq d_{t}\left(f^{n}\right)=\lambda^{n k}$ becomes worse as the dimension grows. Nevertheless, one still has that $d\left(f^{n}, p\right)=O\left((\lambda-1)^{n}\right)$ for a "very generic" choice of $f$ (i.e. for $f$ outside a countable union of hypersurfaces), so we get the following result of Fornaess and Sibony [9].

Corollary 2.3. - Let $f: \mathbb{P}^{k} \rightarrow \mathbb{P}^{k}$ be a "very generic" holomorphic mapping with $\lambda=\delta_{1}(f) \geq 2$. Then

$$
\lambda^{-n}\left(f^{n}\right)^{*} S \longrightarrow T_{f}
$$

for every positive closed current $S$ of bidegree $(1,1)$ and unit mass on $\mathbb{P}^{k}$. In particular $T_{f}$ is the only $f^{*}$-invariant current.

It turns out that looking at local topological degrees is not sufficient to settle the problem of convergence to $T_{f}$ when $k \geq 2$. Our volume estimates (Theorem 1.2) nevertheless allow us to complete the recent work of Favre and Jonsson [7] in dimension 2.

Theorem 2.4. - Let $f: \mathbb{P}^{2} \rightarrow \mathbb{P}^{2}$ be a holomophic mapping with $\lambda \geq 2$. There exists a (possibly empty) totally invariant algebraic subset $\mathcal{E}_{f}$ of $\mathbb{P}^{2}$ such that if $S$ is a positive closed current of bidegree $(1,1)$ and unit mass on $\mathbb{P}^{k}$, then

$$
\nu\left(S, \mathcal{E}_{f}\right)=0 \Longrightarrow \frac{1}{\lambda^{n}}\left(f^{n}\right)^{*} S \rightarrow T_{f} .
$$

TOME $131-2003-\mathrm{N}^{\mathrm{O}} 3$ 
The set $\mathcal{E}_{f}$ can be decomposed as $\mathcal{E}_{1} \cup \mathcal{E}_{2}$, where $\mathcal{E}_{1}$ is a union of at most three lines and $\mathcal{E}_{2}$ is a finite set. The condition $\nu\left(S, \mathcal{E}_{f}\right)=0$ has to be understood as $\nu(S, p)=0$ for every point $p \in \mathcal{E}_{2}$ and almost every point $p$ of $\mathcal{E}_{1}$. We refer the reader to [7] for a proof. The only new information we provide are sufficient volume estimates near points of $\mathcal{E}_{2}$, without the extra assumption made by Favre and Jonsson that $\mathcal{E}_{2}$ consists of "homogeneous points".

2.2. Some polynomial endomorphisms of $\mathbb{C}^{k}$. - Let $f: \mathbb{C}^{k} \rightarrow \mathbb{C}^{k}$ be a proper polynomial mapping, $f=\left(P_{1}, \ldots, P_{k}\right)$, where the $P_{j}$ 's are polynomials with $\lambda=\max \left(\operatorname{deg} P_{j}\right) \geq 2$. We let $d_{t}(f)$ denote the topological degree of $f$ and shall assume here that $d_{t}(f)<\lambda$. Given $S$ a positive closed current of bidegree $(1,1)$ and unit mass on $\mathbb{P}^{k}$, we thus get

$$
\sup _{p \in \mathbb{C}^{k}} \nu\left(S_{n}, p\right) \leq \frac{\sup _{p \in \mathbb{C}^{k}} d\left(f^{n}, p\right)}{\lambda^{n}} \sup _{p \in \mathbb{C}^{k}} \nu\left(S, f^{n}(p)\right) \leq\left(\frac{d_{t}(f)}{\lambda}\right)^{n} \rightarrow 0,
$$

where $S_{n}:=\lambda^{-n}\left(f^{n}\right)^{*} S$.

We still denote by $f$ the meromorphic extension of $f$ to $\mathbb{P}^{k}=\mathbb{C}^{k} \cup(t=0)$, where $(t=0)$ denotes the hyperplane at infinity. Since $f$ is polynomial (hence holomorphic) in $\mathbb{C}^{k}$, the indeterminacy set $I_{f}$ is located within $(t=0)$. Define by induction

$$
X_{1}:=\overline{f\left((t=0) \backslash I_{f}\right)}, \quad X_{j+1}:=\overline{f\left(X_{j} \backslash I_{f}\right)} .
$$

This is a decreasing sequence of irreducible analytic subsets of $(t=0)$. We denote by $X_{f}:=X_{\ell}$ the limit set, which we assume is non empty (this is equivalent to saying that $f$ is algebraically stable, see [19]).

THEOREM 2.5. - Let $f: \mathbb{C}^{k} \rightarrow \mathbb{C}^{k}$ be an algebraically stable polynomial endomorphism with $d_{t}(f)<\lambda=\delta_{1}(f)$. Assume $I_{f}$ is an $f^{-1}$-attracting set and there exists an invariant probability measure $\mu$ such that $\log \left|J_{F S}(f)\right| \in L^{1}(\mu)$. Let $S$ be a positive closed current of bidegree $(1,1)$ and unit mass on $\mathbb{P}^{k}$. If $\nu(S, p)=0$ for all $p \in X_{f}$, then

$$
S_{n}:=\frac{1}{\lambda^{n}}\left(f^{n}\right)^{*} S \longrightarrow T_{f} .
$$

When $\operatorname{dim} X_{f}=0, T_{f}$ is the only $f^{*}$-invariant current of unit mass in $\mathbb{C}^{k}$.

Proof. - We assume $I_{f}$ is $f^{-1}$-attracting in the following sense: there exists an open neighborhood $V$ of $I_{f}$ in $\mathbb{C}^{k}$ such that $\bigcap_{j \geq 0} f^{-j}(V)=\varnothing$. This implies that $f$ is a normal mapping. Note also that $f$ is proper in $\mathbb{C}^{k}$ : if an algebraic curve $\mathcal{C} \subset \mathbb{C}^{k}$ were to be contracted to a point by $f, \mathcal{C}$ would intersect $(t=0)$ within $I_{f}$, contradicting that $I_{f}$ is $f^{-1}$-attracting. Therefore $\sup _{p \in \mathbb{C}^{k}} \nu\left(S_{n}, p\right) \rightarrow 0$ as observed above. If $q \in(t=0) \backslash I_{f^{\ell}}$ then

$$
\nu\left(\left(f^{\ell}\right)^{*} S, q\right) \leq C_{f, q} \nu\left(S, f^{\ell}(q)\right)=0 \text {, since } f^{\ell}(q) \in X_{f}=X_{\ell} .
$$

BULlETiN DE LA SOCIÉtÉ MATHÉMATIQUE DE FRANCE 
The latter inequality is due to Favre [4] and Kiselman [16]. Therefore $\sup _{p \in \mathbb{P}^{k} \backslash I_{f} \ell} \nu\left(S_{n}, p\right) \rightarrow 0$ so $S_{n} \rightarrow T_{f}$ by Theorem 1.4 .

When $\operatorname{dim} X_{f}=0, X_{f}$ is reduced to a point which does not belong to $I_{f}$. In this case $\nu\left(S, X_{f}\right)=0$ is also a necessary condition to get $S_{n} \rightarrow T_{f}$. Indeed if $\nu\left(S, X_{f}\right)=\gamma>0$ then for all $q \in(t=0) \backslash I_{f^{\ell}}$,

$$
\nu\left(\left(f^{\ell}\right)^{*} S, q\right) \geq \nu\left(S, f^{\ell}(q)\right)=\gamma>0 \quad \text { so } \quad\left(f^{\ell}\right)^{*} S \geq \gamma[t=0]
$$

hence $S_{n}$ does not converge to $T_{f}$. In particular if $S$ is $f^{*}$-invariant and does not charge the hyperplane at infinity then $\nu\left(S, X_{f}\right)=0$ hence $S=S_{n} \rightarrow T_{f}$, so $S=T_{f}$. Therefore every $f^{*}$-invariant current is a linear combination of $T_{f}$ and $[t=0]$.

REMARK 2.6. - The previous result applies e.g. to mappings

$$
f=f_{1} \circ \cdots \circ f_{s}, \quad f_{j}:(z, w) \in \mathbb{C}^{2} \mapsto\left(P_{j}(w), Q_{j}(z)+R_{j}(w)\right) \in \mathbb{C}^{2},
$$

where $P_{j}, Q_{j}, R_{j}$ are polynomials of degree $p_{j}, q_{j}, \lambda_{j}$ with $p_{j} q_{j}<\lambda_{j}$. One gets here

$$
d_{t}(f)=\prod_{j=1}^{s} p_{j} q_{j} \quad \text { and } \quad \lambda:=\delta_{1}(f)=\prod_{j=1}^{s} \lambda_{j}>d_{t}(f) .
$$

The set $I_{f}=[1: 0: 0]$ is $f^{-1}$-attracting (see Example 4.1 in [12]) and $X_{f}=$ $[0: 1: 0]$. An invariant measure $\mu$ such that $\log \left|J_{F S}(f)\right| \in L^{1}(\mu)$ is constructed in [12] (Theorem 5.3). Note that when $p_{j}=q_{j}=1$ then $f$ is a complex Hénon mapping. A different proof of Theorem 2.5 was given in this case in the paper [6], where $f^{*}$-invariant currents are characterized for every birational mapping of $\mathbb{C}^{2}$.

We now consider the case of polynomial automorphisms of $\mathbb{C}^{k}$. We can prove finer volume estimates on the set of points whose orbit accumulates $I_{f}$ so that it is not necessary to assume $f$ is normal.

TheOREM 2.7. - Let $f: \mathbb{C}^{k} \rightarrow \mathbb{C}^{k}$ be an algebraically stable polynomial automorphism of $\mathbb{C}^{k}$ such that $X_{f}$ is an $f$-attracting set. Let $S$ be a positive closed current of bidegree $(1,1)$ and unit mass on $\mathbb{P}^{k}$. If $\nu(S, p)=0$ for all $p \in X_{f}$, then

$$
S_{n}:=\frac{1}{\lambda^{n}}\left(f^{n}\right)^{*} S \longrightarrow T_{f}
$$

If $\operatorname{dim} X_{f}=0$ then $T_{f}$ is the only $f^{*}$-invariant current of unit mass in $\mathbb{C}^{k}$.

Proof. - As observed in Remark 1.3, it is not necessary to assume the existence of an invariant measure $\mu$ such that $\log \left|J_{F S}(f)\right| \in L^{1}(\mu)$. We use instead the fact that the euclidean jacobian of $f$ is constant in $\mathbb{C}^{k}$ to derive a lower bound on $\int_{B} \log \left|J_{F S}\left(f^{n}\right)\right| \omega^{k}$. 
We let $B\left(X_{f}\right)$ denote the basin of attraction of $X_{f}$ in $\mathbb{C}^{k}$ and set

$$
\mathcal{K}_{f}:=\mathbb{C}^{k} \backslash B\left(X_{f}\right) .
$$

Since $f$ is proper in $\mathbb{C}^{k}$ with $d_{t}(f)=1<\lambda$, we get as before

$$
\sup _{p \in \mathbb{P}^{k} \backslash I_{f} \ell} \nu\left(S_{n}, p\right) \longrightarrow 0 .
$$

Therefore $S_{n} \rightarrow 0$ in $B\left(X_{f}\right)$. Now we must make sure that $S_{n} \rightarrow 0$ on any relatively compact open subset $B$ of $\mathcal{K}_{f}$ (if any). Write

$$
S=\omega+\operatorname{dd}^{c} u,
$$

where $u \leq 0, u \in L^{1}\left(\mathbb{P}^{k}\right)$. We need to prove that for all $\varepsilon>0$,

$$
\operatorname{vol}\left(B \cap\left\{u_{n}<-\varepsilon\right\}\right) \longrightarrow 0,
$$

where $u_{n}=\lambda^{-n} u \circ f^{n}$. Now $f^{n}\left(B \cap\left\{u_{n}<-\varepsilon\right\}\right) \subset\left\{u<-\varepsilon \lambda^{n}\right\}$ has volume $\leq C_{1} \exp \left(-\varepsilon \lambda^{n}\right)$ because the Lelong numbers of $S_{n}$ are bounded from above by 1 . On the other hand

$$
\begin{aligned}
\operatorname{vol}\left(f^{n}\left(B \cap\left\{u_{n}<-\varepsilon\right\}\right)\right) & =\int_{B \cap\left\{u_{n}<-\varepsilon\right\}}\left|J_{F S}\left(f^{n}\right)\right|^{2} \omega^{k} \\
& =|a|^{2 n} \int_{B \cap\left\{u_{n}<-\varepsilon\right\}}\left(\frac{1+\|z\|^{2}}{1+\left\|f^{n}(z)\right\|^{2}}\right)^{k+1} \omega^{k} \\
& \geq C_{B}^{(\lambda-1)^{n}} \operatorname{vol}\left(B \cap\left\{u_{n}<-\varepsilon\right\}\right),
\end{aligned}
$$

where $|a|:=\left|J_{\text {eucl }}(f)\right|>0$. The latter inequality follows from the fact that $f$ has slow growth on $\mathcal{K}_{f}$ (see below). This yields $\operatorname{vol}\left(B \cap\left\{u_{n}<-\varepsilon\right\}\right) \leq \alpha_{\varepsilon}^{\lambda^{n}}$ where $0<\alpha_{\varepsilon}<1$ so that $u_{n} \rightarrow 0$ on $\mathcal{K}_{f}$.

We finally have to prove that $f$ does not grow too fast on $\mathcal{K}_{f}$. We can write $f=P+Q$, where $P$ is a homogeneous mapping of degree $\lambda$ and $Q$ is a polynomial mapping of degree $\leq \lambda-1$. Identifying $(t=0)$ with $\mathbb{P}^{k-1}$, we can assume $P(t=0)=X_{f}$. Fix $z_{0} \in \mathcal{K}_{f}$ and $z_{n}=f^{n}\left(z_{0}\right)$. We can assume $z_{n}$ accumulates only at $I_{f} \backslash V$, where $V$ is a small neighborhood of $X_{f}$. Define

$$
\zeta_{n}=P\left(z_{n-1}\right), \quad \zeta_{n}^{\prime}=Q\left(z_{n-1}\right),
$$

so $z_{n}=\zeta_{n}+\zeta_{n}^{\prime}$. There is a constant $C>0$ such that

$$
\left|\zeta_{n}\right|_{+} \leq C\left|\zeta_{n}^{\prime}\right|_{+}
$$

where $|p|_{+}:=\max (\|p\|, 1)$. If not then for a subsequence $\left|\zeta_{n}\right| /\left|\zeta_{n}^{\prime}\right| \rightarrow \infty$, so

$$
\frac{z_{n}}{\left\|z_{n}\right\|}=\frac{\zeta_{n}+\zeta_{n}^{\prime}}{\left\|z_{n}\right\|}=\frac{\zeta_{n}}{\left\|\zeta_{n}\right\|}+o(1)=\frac{P\left(z_{n-1}\right)}{\left\|P\left(z_{n-1}\right)\right\|}+o(1) \text {. }
$$

Hence $z_{n}$ converges to $X_{f}$, contradicting our assumption $z_{0} \in \mathcal{K}_{f}$. We infer $\left|\zeta_{n+1}^{\prime}\right|_{+}=\left|Q\left(z_{n-1}\right)\right|_{+} \leq C_{1}\left|z_{n-1}\right|_{+}^{\lambda-1} \leq C_{2}\left|\zeta_{n-1}^{\prime}\right|_{+}^{\lambda-1}$, hence $\left|f^{n}(p)\right|_{+} \leq C_{2}^{\prime}|p|_{+}^{(\lambda-1)^{n}}$ on $\mathcal{K}_{f}$. 
REMARK 2.8. - When $f$ is a "weakly regular" polynomial automorphism (i.e. when $X_{f} \cap I_{f}=\varnothing$, see [13]) then a necessary and sufficient condition to get convergence to $T_{f}$ is that $\sup _{p \in X_{f}} \nu\left(S_{n}, p\right) \rightarrow 0$. This condition can be understood in terms of the local topological degrees of $f_{0}:=f_{\mid X_{f}}$ which is then an holomorphic endomorphism of $X_{f}$. Note that $X_{f}$ may be singular but $X_{f} \simeq \mathbb{P}^{r}$ if it is smooth, so we are back to the situation described in Section 2.1!

For a "generic" $f, f_{0}$ will have no exceptional set so that $S_{n} \rightarrow T_{f}$ iff $\nu\left(S, X_{f}\right)=\inf _{p \in X_{f}} \nu(S, p)=0$. In this generic situation, $T_{f}$ is the only $f^{*}$ invariant current of unit mass in $\mathbb{C}^{k}$ and $\lambda^{-n}\left(f^{n}\right)^{*}[H] \rightarrow T_{f}$ for every hyperplane $H \in\left(\mathbb{P}^{k}\right)^{*} \backslash \mathcal{E}_{f}$, where

$$
\mathcal{E}_{f}:=\left\{H \in\left(\mathbb{P}^{k}\right)^{*} ; X_{f} \subset H\right\}
$$

is an algebraic subset of $\left(\mathbb{P}^{k}\right)^{*}$.

\section{Appendix}

For the reader's convenience we recall here some compactness criteria for families of quasiplurisubharmonic functions which play a central role in this note. We refer the reader to [20] for a systematic discussion of similar results.

LEMMA 3.1. - Let $\left(\varphi_{n}\right)$ be a sequence of plurisubharmonic functions in some connected open subset $\Omega$ of a complex manifold $X$. If $\left(\varphi_{n}\right)$ is locally uniformly bounded from above in $\Omega$, then either $\left(\varphi_{n}\right)$ uniformly converges to $-\infty$, or it is relatively compact for the $L_{\mathrm{loc}}^{1}(\Omega)$-topology.

We refer the reader to [14] (Theorem 3.2.12) for a proof. There is no plurisubharmonic function on $\mathbb{P}^{k}$ (except constants). However there are plenty of quasiplurisubharmonic functions: these are functions $\varphi$ which are locally the sum of a psh and a smooth function, so that their curvature is allowed to be negative but with a smooth control: $\operatorname{dd}^{c} \varphi \geq-\theta$, where $\theta$ is a smooth form. Of particular interest for us is the following class

$$
\mathcal{L}_{\omega}:=\left\{\varphi \in L^{1}\left(\mathbb{P}^{k}\right) ; \varphi \text { is u.s.c. and } \operatorname{dd}^{c} \varphi \geq-\omega\right\},
$$

where $\omega$ denotes as usual the Fubini-Study Kähler form on $\mathbb{P}^{k}$. Lemma 3.1 easily extends to qpsh functions whose curvature is bounded below by a fixed form, for instance $-\omega$. We make constant use of the following criteria which tell that $\mathcal{L}_{\omega}$ is a compact family once normalized.

Proposition 3.2. - Let $B$ be an open subset of $\mathbb{P}^{k}$. Then

$$
\mathcal{F}_{1}:=\left\{\varphi \in \mathcal{L}_{\omega} ; \sup _{B} \varphi=0\right\} \text { and } \mathcal{F}_{2}:=\left\{\varphi \in \mathcal{L}_{\omega} ; \int_{B} \varphi \omega^{k}=0\right\}
$$

are compact families of qpsh functions.

TOME $131-2003-\mathrm{N}^{\mathrm{O}} 3$ 
Proof. - Fix $B^{\prime} \subset B$ a small ball. We can assume $B^{\prime}=B(r)$ is the ball of radius $r$ centered at the origin in some affine chart $\mathbb{C}^{k} \subset \mathbb{P}^{k}$.

Let $\varphi \in \mathcal{F}_{1}$. Then

$$
\psi:=\varphi+\frac{1}{2} \log \left[1+\|z\|^{2}\right]
$$

is a psh function with logarithmic growth in $\mathbb{C}^{k}$ (because $\omega=\mathrm{dd}^{c}\left(\frac{1}{2} \log [1+\right.$ $\left.\left.\|z\|^{2}\right]\right)$ in $\left.\mathbb{C}^{k}\right)$. Moreover $\sup _{B^{\prime}} \psi \leq \sup _{B^{\prime}} \varphi+\frac{1}{2} \log \left[1+r^{2}\right] \leq \frac{1}{2} \log \left[1+r^{2}\right]$. Therefore

$$
\psi(z) \leq u_{r}(z):=\max \left(\log \frac{\|z\|}{r}, 0\right)+\frac{1}{2} \log \left[1+r^{2}\right] \text { in } \mathbb{C}^{k} .
$$

Indeed one can check this on any line $L \simeq \mathbb{C}$ passing through the origin: the function $u_{r \mid L}$ is harmonic outside the disk $L \cap B(r)$ and dominates $\psi_{\mid L}$ on $\partial(L \cap B(r))$ and near infinity. This yields

$$
\varphi \leq \max \left(\log \frac{\|z\|}{r}, 0\right)+\frac{1}{2} \log \left[1+r^{2}\right]-\frac{1}{2} \log \left[1+\|z\|^{2}\right] \leq c_{r} \text { in } \mathbb{C}^{k},
$$

hence in $\mathbb{P}^{k}$. Therefore functions of $\mathcal{F}_{1}$ are uniformly bounded from above on $\mathbb{P}^{k}$. So if we fix a sequence $\left(\varphi_{j}\right)$ of functions in $\mathcal{F}_{1}$, we can extract a convergent subsequence by Lemma 3.1. The cluster point is not $-\infty$ because $\sup _{B} \varphi_{j}=0$ hence it belongs to $\mathcal{F}_{1}$.

The argument is similar for $\mathcal{F}_{2}$. We simply need to derive a uniform upper bound on $\mathbb{P}^{k}$. So let $\varphi \in \mathcal{F}_{2}$. Then $\varphi-\sup _{B} \varphi \in \mathcal{F}_{1}$ which is compact. Therefore there exists $C$ such that

$$
\int_{B}\left|\varphi-\sup _{B} \varphi\right| \omega^{k} \leq C, \text { for all } \varphi \in \mathcal{F}_{2} .
$$

This yields $\sup _{B} \varphi \leq C \operatorname{vol}(B)$ since $\int_{B} \varphi \omega^{k}=0$. Moreover there exists $C^{\prime}>0$ such that $\sup _{\mathbb{P} k} \psi \leq C^{\prime}$ for every $\psi \in \mathcal{F}_{1}$, so

$$
\varphi=\left(\varphi-\sup _{B} \varphi\right)+\sup _{B} \varphi \leq C^{\prime}+C \operatorname{vol}(B) \text { on } \mathbb{P}^{k},
$$

for every $\varphi \in \mathcal{F}_{2}$. This implies the compactness of $\mathcal{F}_{2}$.

\section{BIBLIOGRAPHY}

[1] Bedford (E.) \& Smillie (J.) - Polynomial diffeomorphisms of $\mathbb{C}^{2}$ : currents, equilibrium measure and hyperbolicity, Invent. Math., t. 103 (1991), no. 1, pp. 69-99.

[2] Berteloot (F.) \& Mayer (V.) - Rudiments de dynamique holomorphe, Cours spécialisés, vol. 7, Société Mathématique de France, Paris, 2001.

[3] Brolin (H.) - Invariant sets under iteration of rational functions, Arkiv. Math., t. 6 (1965), pp. 103-144. 
[4] FAvre (C.) - Note on pull-back and Lelong number of currents, Bull. Soc. Math. France, t. 127 (1999), no. 3, pp. 445-458.

[5] Multiplicity of holomorphic functions, Math. Ann., t. 316 (2000), pp. $355-378$.

[6] Favre (C.) \& Guedu (V.) - Dynamique des applications rationnelles des espaces multiprojectifs, Indiana Univ. Math. J., t. 50 (2001), pp. 881-934.

[7] Favre (C.) \& Jonsson (M.) - Brolin Theorem for curves in two complex dimensions, Preprint, 2001.

[8] Fornaess (J.-E.) \& Sibony (N.) - Complex Hénon mappings in $\mathbb{C}^{2}$ and Fatou-Bieberbach domains, Duke Math. J., t. 65 (1992), no. 2, pp. 345-380.

[9] _ Complex dynamics in higher dimension II, in Modern methods in complex analysis (Princeton, NJ, 1992), Ann. of Math. Stud., vol. 137, Princeton Univ. Press, Princeton, NJ, 1995, pp. 135-182.

[10] Freire (A.), Lopes (A.) \& MaÑé (R.) - An invariant measure for rational maps, Bol. Soc. Brasil Mat., t. 14 (1983), pp. 45-62.

[11] Griffiths (P.A.) \& Harris (J.) - Principles of algebraic geometry, Wiley, New York, 1978.

[12] Guedu (V.) - Dynamics of polynomial mappings of $\mathbb{C}^{2}$, Amer. J. Math., t. 124 (2002), pp. 75-106.

[13] Guedu (V.) \& Sibony (N.) - Dynamics of polynomial automorphisms of $\mathbb{C}^{k}$, Arkiv. Math., t. 40 (2002), pp. 207-243.

[14] Hörmander (L.) - Notions of convexity, Progress in Math., vol. 127, Birkhäuser Boston, Inc., Boston, MA, 1994.

[15] Hubbard (J.H.) \& Papadopol (P.) - Superattractive fixed points in $\mathbb{C}^{n}$, Indiana Univ. Math. J., t. 43 (1994), pp. 321-365.

[16] Kiselman (C.) - Ensembles de sous-niveau et images inverses des fonctions plurisousharmoniques, Bull. Sci. Math., t. 124 (2000), no. 1, pp. 7592.

[17] LyUBich (M.Ju.) - Entropy properties of rational endomorphisms of the Riemann sphere, Ergodic Theory \& Dyn. Syst., t. 3 (1983), pp. 351-385.

[18] Russakovskit (A.) \& Shiffman (B.) - Value distribution for sequences of rational mappings and complex dynamics, Indiana Univ. Math. J., t. 46 (1997), pp. 897-932.

[19] Sibony (N.) - Dynamique des applications rationnelles de $\mathbb{P}^{k}$, in Dynamique et géométrie complexes, Panoramas et Synthèses, Société Mathématique de France, Paris, 1999, pp. 97-185.

[20] Zeriahi (A.) - A criterion of algebraicity for Lelong classes and analytic sets, Acta Math., t. 184 (2000), no. 1, pp. 113-143. 